\title{
The effectiveness of Kinesio taping on pain and clinical features in chronic non-specific low back pain: A randomized controlled clinical trial
}

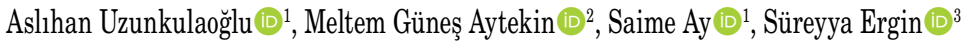 \\ ${ }^{1}$ Department of Physical Medicine and Rehabilitation, Ufuk University Faculty of Medicine, Ankara, Turkey \\ ${ }^{2}$ Department of Physical Medicine and Rehabilitation, Ankara Physical Medicine and Rehabilitation Training and Research Hospital, Ankara, Turkey \\ ${ }^{3}$ Department of Physical Medicine and Rehabilitation, Fizyocare Physical Medicine and Rehabilitation Center, Ankara, Turkey
}

Received: June 20, 2017 Accepted: September 27, 2017 Published online: May 15, 2018

\begin{abstract}
Objectives: This study aims to investigate the effectiveness of Kinesio taping (KT) and sham KT on pain, lumbar range of motion (ROM) and disability in chronic non-specific low back pain.

Patients and methods: This is a randomized, placebo controlled study. Sixty patients ( 22 males, 38 females; mean age $21.5 \pm 1.7$ years, range, 19 to 25 years) with chronic low back pain were randomized into either intervention or placebo groups. Group 1 ( $\mathrm{n}=30$ ) was treated with KT and group $2(n=30)$ was treated with sham taping six times by intervals of three days. Patients were evaluated according to pain, modified Schober's test value, hand-ground distance and lumbar ROM and disability at baseline and at the end of the first and sixth months of intervention. Pain was assessed by using Visual Analog Scale (VAS), while ROM was assessed by modified Schober's test value, hand-ground distance and lumbar lateral flexion ROM. Disability was assessed with Oswestry Disability Index (ODI).

Results: Statistically significant improvements for all parameters were found for both groups after one month of intervention ( $<<0.05)$. These effects were sustained after six months of follow-up for modified Schober's test, hand-ground distance and ODI. There were statistically significant differences between the groups for all outcome measures at the first month of intervention $(\mathrm{p}<0.05)$. However, at sixth-month follow-up, only modified Schober's test, hand-ground distance and ODI values were significantly different between groups $(\mathrm{p}<0.05)$ except VAS ( $\mathrm{p}>0.05)$.

Conclusion: Kinesio taping provided significant improvements in pain, ROM and disability at short term. These positive effects were sustained for ROM and disability at long term but not for pain.
\end{abstract}

Keywords: Chronic non-specific low back pain; disability; Kinesio taping.

Chronic low back pain is a common musculoskeletal disorder associated with enormous health and socioeconomic costs. ${ }^{[1-4]}$ Also, this condition causes restriction on mobility, disability and impairment in quality of life. ${ }^{[5,6]}$ Being pain-free likelihood for 12 months after the onset of chronic low back pain is only $42 \% .{ }^{[7]}$ Because of this reason, there is an urgent need for more effective treatments for this condition.

The current literature provides several possibilities for the treatment of low back pain like educational programs, behavioral cognitive therapy, medication, electrophysical agents, manual therapy and general exercises. ${ }^{[8]}$ A relatively new treatment method for low back pain is Kinesio taping (KT), which is being widely used. ${ }^{[8]}$

Kinesio taping is an elastic bandage method of which the mechanism is not clearly understood. This method was developed by Kenzo Kase in 1970. This elastic bandage is thinner and more elastic than conventional tape, so it allows greater skin traction and mobility. ${ }^{[9]}$ This special material is waterproof but non-degradable in water. This tape can be stretched to $140 \%$ of its original length, thus producing less mechanical retention and restriction for movement. ${ }^{[10]}$ The therapist decides what level of tension will generate an appropriate level of traction

Corresponding author: Aslıhan Uzunkulaoğlu, MD. Ufuk Üniversitesi Tıp Fakültesi Dr. Rıdvan Ege Hastanesi Fiziksel Tıp ve Rehabilitasyon Anabilim Dalı, 06520 Balgat, Ankara, Turkey. e-mail: aslihanseyrek@gmail.com 
on the skin. With this traction, an elevation of the epidermis can be promoted and then it reduces the pressure on the mechanoreceptors, which are located below the dermis. Besides, the nociceptive stimulus is reduced with this mechanism. The studies suggest that the tape can improve blood and lymphatic circulation, reduces pain, realigns joints and reduces muscle tension. ${ }^{[11,12]}$ There are many mechanisms for explaining the effects of KT. In a study, KT applied to lumbar muscles influenced chronic low back pain patients clinically and electromyographically. ${ }^{[13]}$ Although $\mathrm{KT}$ acts on pain are not yet clear, some hypotheses had been made until now. ${ }^{[13]}$ Sensory modalities act within interconnecting, intermodal and crossmodal networks. ${ }^{[14]}$ Furthermore, some studies have suggested that keratinocytes may represent the nonneuronal primary transducers of mechanical stimuli and probably through signal transduction cascade mechanisms such as intracellular calcium ion fluxes to evoke a response in adjacent $\mathrm{C}$-fibers. ${ }^{[15,16]}$ On the other hand, KT may provide afferent stimuli and facilitate pain inhibitory mechanisms and pain reduction. ${ }^{[17]}$

Kinesio taping is also likely to change the pattern of recruitment of muscle fibers. ${ }^{[12,18]}$ Still, this type of treatment has to be scientifically investigated since randomized controlled studies are limited. Therefore, in this study, we aimed to investigate the effectiveness of KT and sham KT on pain, lumbar range of motion (ROM) and disability in chronic non-specific low back pain.

\section{PATIENTS AND METHODS}

\section{Study design}

This randomized, placebo controlled trial with a blind assessor was conducted in Ufuk University Hospital between May 2015 and December 2015. The study protocol was approved by the Institutional Review Board of the Ufuk University Ethics Committee (The registration number for the study is 30042015-4). A written informed consent was obtained from each patient. The study was conducted in accordance with the principles of the Declaration of Helsinki.

\section{Participants}

Power and Sample Size Software (PASS; NCSS, Utah, USA) was used to calculate the sample size taking into account the difference between the Oswestry Disability Index (ODI) means (5 units) and standard deviation (6 units) at first week and alpha error $(p<0.05)$ between the intervention group and the placebo group. ${ }^{[19,20]}$ If the true difference between the means of the intervention and placebo groups is 5 , we will need to study 24 intervention subjects and 24 placebo subjects to be able to reject the null hypothesis that the population means of the intervention and placebo groups are equal with probability (power) of 0.8 . The Type I error probability associated with this test of this null hypothesis is 0.05 . To allow for at least $10 \%$ loss to follow-up, we increased the sample size to 60 .

Sixty patients (22 males, 38 females; mean age $21.5 \pm 1.7$ years, range, 19 to 25 years) whom diagnosed as chronic non-specific low back pain and with normal full blood count, erythrocyte sedimentation rate (ESR), $\mathrm{C}$-reactive protein (CRP) and biochemical markers were recruited. Chronic non-specific low back pain is defined as a primary complaint of pain in the area between the $12^{\text {th }}$ rib and buttock crease for more than 12 -week duration. Individuals were considered of inclusion if they were between 18-30 years and had pain with minimum scoring of 3 on a Visual Analog Scale (VAS) for pain intensity. Individuals were excluded if they had presence of skin diseases; contraindication due to use of the tape, pregnancy, known or suspected serious congenital spinal pathology, spinal surgery history, lumbar disc herniation, rheumatoid arthritis or spondyloarthropathy diagnosis; and physical therapy for low back pain during the previous six months.

By using the method on the website http://randomizer.org/ (Social Physiology Network, Middletown, Connecticut), participants were randomly assigned into two as either intervention $(K T, n=30)$ or placebo $(\mathrm{n}=30)$ groups. $^{[21]}$ All patients were blinded to treatment allocation while the physiotherapist who applied the therapy was aware of the procedure. Also, the physiatrist who performed the examination and evaluations were blinded to treatment allocation.

\section{Intervention and placebo groups}

Intervention group $(\mathrm{n}=30)$ was treated with Kinesio tape (Kinesio Tex Gold, 2in x 103.3ft, Kinesio ${ }^{\circledR}$, Albuquerque, New Mexico, USA) suggested by Kase et al. ${ }^{[10]}$ six times by intervals of three days for 15 days. After the physiatrist's evaluation, a certified physiotherapist for this method applied the taping. Taping was begun by placing the patient in a neutral spine position and then the base of Kinesio Y strip was applied in the sacroiliac joint region, a minimum of $5 \mathrm{~cm}$ below the initiation of pain. For application of the tail of the Y strip on the right side, patients were asked to move into flexion with rotation to opposite side. The tail was applied with very light to light tension (15-25\% of available) or paper-off tension. For the last approximately $5 \mathrm{~cm}$, physiotherapist laid down the tail 
with no tension. Either the patient was returned to neutral position, or moved into forward flexion with rotation to the opposite side (Figure 1). So, the second Kinesio Y tail would be properly applied (Figure 2). ${ }^{[10]}$

Placebo group $(n=30)$ was treated with sham taping six times by intervals of three days for 15 days. Sham taping was applied with a "Y" strip of the same material on ineffective parts of the muscle without a tension to sacroiliac joint in neutral position (Figure 3).

A home-based exercise program including isometric-isotonic lumbar exercises and back extensor stretching exercises were given to all patients every day during the treatment. No medications including analgesic drugs or non-steroidal anti-inflammatory drugs were allowed during the treatment process. Patients were told to avoid uncontrolled or vigorous activities. Also, they were advised to remain active as tolerated and to return to their normal daily activities. Patients were instructed to keep the tape on between taping sessions.

\section{Outcome measures}

Patients were evaluated at baseline, at the end of the first month intervention and at the end of sixmonth follow-up. The primary outcome measure of the study was pain intensity and the secondary outcome measures of the study were modified Schober's test value, hand-ground distance, lumbar lateral flexion ROM for both right and left sides and the ODI.

\section{Pain intensity}

Pain intensity was measured on VAS, where $0=$ no pain and $10=$ worst possible pain. This scale was completed by the patients.

\section{Modified Schober's test value}

Patients were standing with their back towards the examiner. The examiner determined the location of the lumbosacral junction by precising the location of the dimples of Venus. The intersection of the top of the dimples of Venus is marked by drawing a horizontal line. This line acts as the landmark. The second line was marked $10 \mathrm{~cm}$ above the first and the third was marked $5 \mathrm{~cm}$ below the first line. The difference between the measurements in erect and flexion positions indicates the outcome of the lumbar flexion. ${ }^{[22]}$

\section{Hand-Ground distance}

Patients were asked to bend forward and try to touch the floor with fingers. The remaining distance between third finger and ground was measured (centimeters).

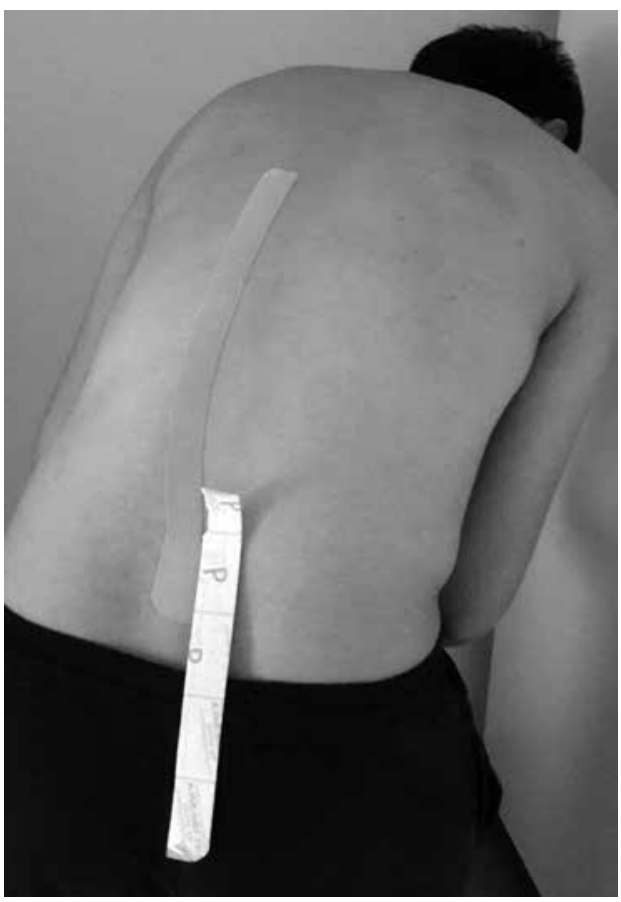

Figure 1. Kinesio taping application (while patient turning to opposite side).

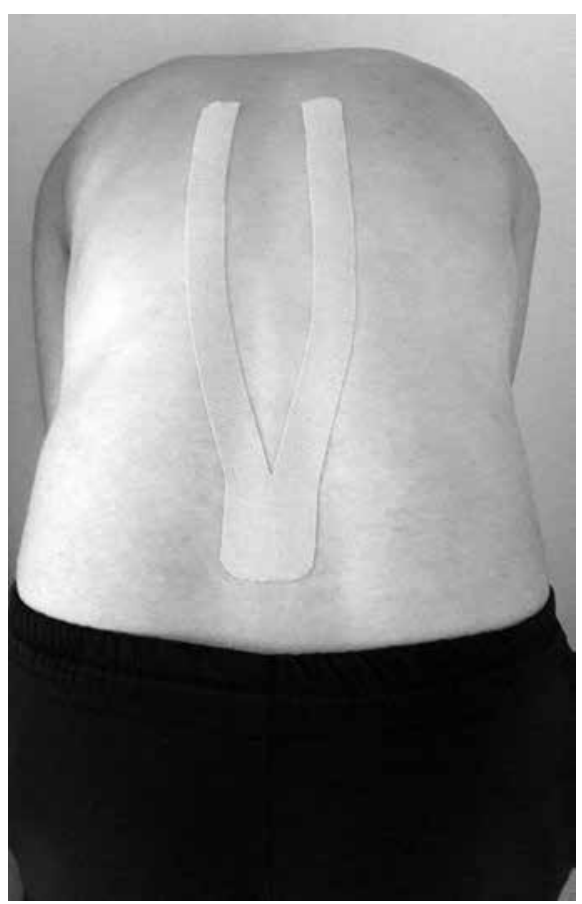

Figure 2. Kinesio taping application (when second Kinesio Y tail would be applied). 


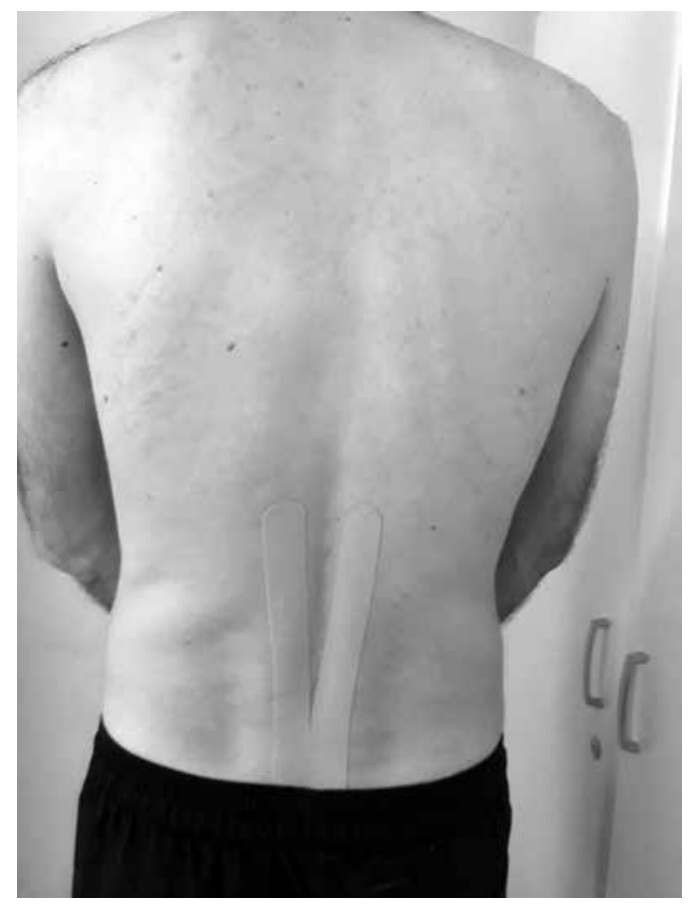

Figure 3. Placebo Kinesio taping application (with no tension).

\section{Lumbar lateral flexion range of motion}

The active lumbar ROM (right-left lateral flexion) was measured using a goniometer when the patient was standing in neutral position.

\section{The Oswestry Disability Index}

The ODI is a self-rating questionnaire used to evaluate functional physical disability. It includes 10 sections of six propositions; each rated on a $0-5$ scale. Relative values are reported (total score/ total possible score $\times 100 \%)$. Higher scores indicate worse disability. ${ }^{[23]}$

\section{Statistical analysis}

The means and standard deviations were given as descriptive statistics. All data for normality were tested by using Kolmogorov-Smirnov test. Paired-samples t-test was used to calculate the pre- and post-treatment value differences. To compare the differences between two groups, the Student's t-test was used. A level of significance of $\mathrm{p}<0.05$ was accepted. All analyses were performed using the PASW for Windows 18.0 software program (SPSS Inc., Chicago, IL, USA).

\section{RESULTS}

All 60 of the participants completed the study protocol and none of participants had any allergic reactions or any other side effects.

The demographic characteristics and baseline values of the outcome measures of the patients are presented in Table 1 . No statistically significant differences were detected between the groups at baseline values $(p>0.05)$ except age, modified Schober's test and lumbar left lateral flexion $\mathrm{ROM}$ values $(\mathrm{p}<0.05)$ (Table 1$)$.

For both intervention and placebo groups, statistically significant improvement in VAS $(\mathrm{p}=0.000)$, modified Schober's test $(\mathrm{p}=0.000)$, handground distance $(\mathrm{p}=0.000$ and $\mathrm{p}=0.038$, respectively), right lumbar lateral flexion ROM $(\mathrm{p}=0.000)$ and ODI values $(p=0.000)$ were found after one month of intervention. These effects were sustained after six months of follow-up for both intervention and placebo groups for modified Schober's test $(\mathrm{p}=0.000)$, handground distance $(\mathrm{p}=0.000$ and $\mathrm{p}=0.019$, respectively) and ODI ( $p=0.000$ and $p=0.008$, respectively) but not for VAS ( $\mathrm{p}=0.136$ and $\mathrm{p}=1.000$, respectively) and right lumbar lateral flexion $\operatorname{ROM}(\mathrm{p}=0.129$ and $\mathrm{p}=0.211$, respectively) (Table 2).

Table 1. Demographic characteristics and baseline values of outcome measures

\begin{tabular}{|c|c|c|c|c|c|}
\hline \multirow[t]{2}{*}{ Variable } & \multicolumn{2}{|c|}{ Intervention group $(\mathrm{n}=30)$} & \multicolumn{2}{|c|}{ Placebo group $(n=30)$} & \multirow[b]{2}{*}{$p$} \\
\hline & $\mathrm{n}$ & Mean \pm SD & $\mathrm{n}$ & Mean \pm SD & \\
\hline Age (year) & & $21.63 \pm 1.771$ & & $21.27 \pm 1.617$ & $0.000^{\star}$ \\
\hline Gender & & & & & 0.206 \\
\hline Female & 19 & & 19 & & \\
\hline Male & 11 & & 11 & & \\
\hline Visual Analog Scale & & $5.97 \pm 1.299$ & & $5.80 \pm 1.627$ & 0.142 \\
\hline Modified Schober's test & & $20.40 \pm 1.070$ & & $19.10 \pm 0.923$ & $0.048^{*}$ \\
\hline Hand-ground distance & & $14.87 \pm 10.082$ & & $8.13 \pm 3.309$ & 0.213 \\
\hline Lumbar lateral flexion ROM (right) & & $15.63 \pm 3.200$ & & $13.07 \pm 1.363$ & 0.099 \\
\hline Lumbar lateral flexion ROM (left) & & $13.00 \pm 2.792$ & & $13.27 \pm 1.172$ & $0.024^{*}$ \\
\hline Oswestry Disability Index & & $33.67 \pm 17.389$ & & $22.33 \pm 11.118$ & 0.386 \\
\hline
\end{tabular}

SD: Standard deviation; ${ }^{\star} \mathrm{p}<0.05$. ROM: Range of motion. 
Table 2. Comparison of outcome measures in both groups and between groups

\begin{tabular}{|c|c|c|c|c|c|}
\hline \multirow[b]{2}{*}{ Variable } & \multicolumn{2}{|c|}{ Intervention group $(n=30)$} & \multicolumn{2}{|c|}{ Placebo group $(n=30)$} & \multirow[b]{2}{*}{$p$} \\
\hline & Mean \pm SD & $p$ & Mean \pm SD & $p$ & \\
\hline \multicolumn{6}{|l|}{ Visual Analog Scale } \\
\hline Baseline & $5.97 \pm 1.299$ & \multirow{2}{*}{$0.000^{*}$} & $5.80 \pm 1.627$ & \multirow{2}{*}{$0.000^{*}$} & \multirow{2}{*}{$0.000^{\star}$} \\
\hline Post-treatment $1^{\text {st }}$ month & $3.23 \pm 1.006$ & & $5.13 \pm 1.408$ & & \\
\hline Follow-up $6^{\text {th }}$ month & $6.16 \pm 1.05$ & 0.136 & $5.80 \pm 1.374$ & 1.000 & 0.251 \\
\hline \multicolumn{6}{|l|}{ Modified Schober's test } \\
\hline Baseline & $19.13 \pm 1.224$ & \multirow{2}{*}{$0.000^{\star}$} & $19.10 \pm 0.923$ & \multirow{2}{*}{$0.000^{*}$} & \multirow{2}{*}{$0.004^{\star}$} \\
\hline Post-treatment $1^{\text {st }}$ month & $20.40 \pm 1.070$ & & $19.63 \pm 0.928$ & & \\
\hline Follow-up $6^{\text {th }}$ month & $20.27 \pm 0.944$ & $0.000^{*}$ & $19.63 \pm 0.890$ & $0.000^{*}$ & $0.010^{\star}$ \\
\hline \multicolumn{6}{|l|}{ Hand-ground distance } \\
\hline Baseline & $12.03 \pm 8.430$ & \multirow{2}{*}{$0.000^{*}$} & $8.13 \pm 3.309$ & \multirow{2}{*}{$0.038^{\star}$} & \multirow{2}{*}{$0.003^{\star}$} \\
\hline Post-treatment $1^{\text {st }}$ month & $14.87 \pm 10.082$ & & $8.70 \pm 3.852$ & & \\
\hline Follow-up $6^{\text {th }}$ month & $14.83 \pm 10.505$ & $0.000^{\star}$ & $8.77 \pm 3.766$ & 0.019 & $0.004^{\star}$ \\
\hline \multicolumn{6}{|c|}{ Lumbar lateral flexion ROM (right) } \\
\hline Baseline & $13.93 \pm 3.248$ & \multirow{2}{*}{$0.000^{\star}$} & $13.07 \pm 1.363$ & \multirow{2}{*}{$0.000^{*}$} & \multirow{2}{*}{0.001} \\
\hline Post-treatment $1^{\text {st }}$ month & $15.63 \pm 3.200$ & & $13.53 \pm 1.106$ & & \\
\hline Follow-up $6^{\text {th }}$ month & $13.70 \pm 3.053$ & 0.129 & $12.93 \pm 1.413$ & 0.211 & 0.217 \\
\hline \multicolumn{6}{|c|}{ Lumbar lateral flexion ROM (left) } \\
\hline Baseline & $13.00 \pm 2.792$ & \multirow{2}{*}{$0.000^{*}$} & $13.27 \pm 1.172$ & \multirow{2}{*}{0.118} & \multirow{2}{*}{$0.007^{\star}$} \\
\hline Post-treatment $1^{\text {st }}$ month & $14.97 \pm 2.553$ & & $13.53 \pm 1.224$ & & \\
\hline Follow-up $6^{\text {th }}$ month & $13.07 \pm 2.664$ & 0.662 & $13.17 \pm 1.440$ & 0.264 & 0.857 \\
\hline \multicolumn{6}{|l|}{ Oswestry Disability Index } \\
\hline Baseline & $33.67 \pm 17.389$ & \multirow{2}{*}{$0.000^{*}$} & $22.33 \pm 11.118$ & \multirow{2}{*}{$0.000^{*}$} & \multirow{2}{*}{$0.045^{\star}$} \\
\hline Post-treatment $1^{\text {st }}$ month & $23.67 \pm 13.956$ & & $17.27 \pm 9.944$ & & \\
\hline Follow-up $6^{\text {th }}$ month & $27.50 \pm 14.545$ & $0.000^{*}$ & $20.07 \pm 9.986$ & 0.008 & $0.025^{\star}$ \\
\hline
\end{tabular}

SD: Standard deviation; ${ }^{*} \mathrm{p}<0.05$. ROM: Range of motion.

One month after the treatment, statistically significant differences were observed in VAS $(p=0.000)$, modified Schober's test $(\mathrm{p}=0.004)$, hand-ground distance ( $\mathrm{p}=0.003)$, right and left lumbar lateral flexion ROM ( $\mathrm{p}=0.001$ and $\mathrm{p}=0.007$, respectively) and ODI $(\mathrm{p}=0.045)$ between the groups (Table 2$)$.

After six months of follow-up, only modified Schober's test $(\mathrm{p}=0.004)$, hand-ground distance $(\mathrm{p}=0.004)$ and ODI $(\mathrm{p}=0.025)$ values were statistically significantly different between groups. However, no differences were found in VAS $(\mathrm{p}=0.251)$ and right and left lumbar lateral flexion ROM ( $\mathrm{p}=0.217$ and $\mathrm{p}=0.857$, respectively (Table 2).

\section{DISCUSSION}

Non-specific chronic low back pain is a common musculoskeletal disorder with a high prevalence and recurrence. ${ }^{[3]}$ Because of pain and disability, personal and socioeconomic burdens can occur. Kinesio taping is an alternative technique used in chronic low back pain. ${ }^{[24]}$ Although this technique was described a long time ago, it has recently become popular. ${ }^{[25]}$ There are limited numbers of randomized controlled studies about the effect of KT for chronic low back pain. ${ }^{[24,26-28]}$ Nelson $^{[29]}$ reviewed the studies which investigate the effects of KT for chronic low back pain and revealed that $\mathrm{KT}$ may be most effective when used as an additional therapy, while higher quality studies highlighted that multiple factors mediate low back pain in the short, intermediate and long term. Because of these reasons, we aimed to determine the short and long term effects of KT for people with chronic non-specific low back pain versus placebo tape application to the lumbar spine in terms of pain and disability.

In a study, the effectiveness of $\mathrm{KT}$ and sham $\mathrm{KT}$ in 60 patients with chronic low back pain were 
compared. Significant improvements in pain intensity and disability measures were reported for KT group at one week; but there was no significant difference at four weeks. ${ }^{[9]}$ In another study by Parreira et al., ${ }^{[24]}$ the effects of KT and sham taping for people with chronic non-specific low back pain were compared and at the end of the treatment protocol, there were no significant differences between groups in terms of pain intensity and disability measures at both four and 12 weeks after randomization. In our study, we determined significant improvement for pain intensity and disability measures at one month of the intervention in KT group and these positive effects were sustained for ROM and disability at long term. As a power of our work, we evaluated the patients in a longer time period and determined statistically significant differences between the groups for disability. This result may be due to the application of the taping method. From this point, our application method of Y strip with a specific tension may have led to an increased sensitivity of cutaneous receptors and produced more facilitation. There is no gold standard for the application method and period for KT for chronic low back pain and the application methods in the mentioned studies were different from our study. Also, we repeated the application six times. Thus, we may conclude that the appropriate application method and frequency for KT is important for the effectiveness of this therapy in terms of disability for both short and long term.

In the literature, there are also studies which investigated the effect of $\mathrm{KT}$ application for acute back pain. ${ }^{[30]}$ In a study by Kelle et al., ${ }^{[30]} 109$ patients with acute non-specific low back pain were included and randomized into either $\mathrm{KT}$ or placebo groups; both groups received merely information and reassurance. KT was applied for 12 days. Patients were evaluated at the end of intervention and at the fourth week. At the end of the study, authors concluded that $\mathrm{KT}$ provided significant improvements in pain and disability. These results are consistent with our study in which we observed significant improvements in pain and disability at one month for chronic non-specific low back pain. The power of our work is that we investigated patients for a longer period of time and compared them to those with sham taping.

In a study conducted by Kachanathu et al., ${ }^{[31]}$ 40 patients with non-specific low back pain were included and randomly divided into two groups; a group underwent conventional physical therapy with KT and the other group underwent only conventional physical therapy. There were significant differences in measures of pain and ROM in each group; however, there was no significant difference between the groups. Furthermore, unlike some other studies in the literature ${ }^{[9,24]}$ we determined statistically significant differences in VAS, modified Schober's test, hand-ground distance, right lumbar lateral flexion ROM and ODI values between groups after one month of intervention. Modified Schober's test, hand-ground distance and ODI values were significant between groups after six months of follow-up. This result can be explained by the exercise program in addition to $\mathrm{KT}$ application in our protocol. Therefore, improvements may be due to the positive effects of exercise. On the other hand, we did not form an only-exercise group that could be compared to intervention and placebo groups and this can be a limitation for our study.

In conclusion, $\mathrm{KT}$ provided significant improvements in pain, ROM and disability in chronic non-specific low back pain at short term. These positive effects were sustained for ROM and disability at long term but not for pain. These results may vary according to application methods and frequency. Since $\mathrm{KT}$ is a non-invasive physical therapy modality and there is no accepted consensus on standard therapy for optimum duration, frequency and application technique, further large studies with larger patient populations are needed to explain the application method.

\section{Declaration of conflicting interests}

The authors declared no conflicts of interest with respect to the authorship and/or publication of this article.

\section{Funding}

The authors received no financial support for the research and/or authorship of this article.

\section{REFERENCES}

1. de Hoyo M, Álvarez-Mesa A, Sañudo B, Carrasco L, Domínguez S. Immediate effect of kinesio taping on muscle response in young elite soccer players. J Sport Rehabil 2013;22:53-8.

2. Dagenais S, Caro J, Haldeman S. A systematic review of low back pain cost of illness studies in the United States and internationally. Spine J 2008;8:8-20.

3. Pengel LH, Herbert RD, Maher CG, Refshauge KM. Acute low back pain: systematic review of its prognosis. BMJ 2003;327:323.

4. Walker BF. The prevalence of low back pain: a systematic review of the literature from 1966 to 1998. J Spinal Disord 2000;13:205-17.

5. Andersson GB. Epidemiological features of chronic lowback pain. Lancet 1999;354:581-5. 
6. Ryan CG, Grant PM, Dall PM, Gray H, Newton M, Granat MH. Individuals with chronic low back pain have a lower level, and an altered pattern, of physical activity compared with matched controls: an observational study. Aust J Physiother 2009;55:53-8.

7. Costa Lda C, Maher CG, McAuley JH, Hancock MJ, Herbert RD, Refshauge KM, et al. Prognosis for patients with chronic low back pain: inception cohort study. BMJ 2009;339:b3829.

8. Airaksinen O, Brox JI, Cedraschi C, Hildebrandt J, KlaberMoffett J, Kovacs F, et al. Chapter 4. European guidelines for the management of chronic nonspecific low back pain. Eur Spine J 2006;15 Suppl 2:S192-300.

9. Castro-Sánchez AM, Lara-Palomo IC, Matarán-Peñarrocha GA, Fernández-Sánchez M, Sánchez-Labraca N, ArroyoMorales M. Kinesio Taping reduces disability and pain slightly in chronic non-specific low back pain: a randomised trial. J Physiother 2012;58:89-95.

10. Kase K, Wallis J, Kase T. Clinical therapeutic applications of the Kinesio taping method. 2nd ed. Tokyo: Ken Ikai Co Ltd; 2003.

11. Kase K, Tatsuyuki H, Tomoki O. Development of Kinesio tape. In: Kinesio Taping Perfect Manual. Albuquerque NM: Kinesio USA Ltd; 1996. p. 117-8.

12. Hsu YH, Chen WY, Lin HC, Wang WT, Shih YF. The effects of taping on scapular kinematics and muscle performance in baseball players with shoulder impingement syndrome. J Electromyogr Kinesiol 2009;19:1092-9.

13. Paoloni M, Bernetti A, Fratocchi G, Mangone M, Parrinello L, Del Pilar Cooper M, et al. Kinesio Taping applied to lumbar muscles influences clinical and electromyographic characteristics in chronic low back pain patients. Eur J Phys Rehabil Med 2011;47:237-44.

14. McGlone F, Reilly D. The cutaneous sensory system. Neurosci Biobehav Rev 2010;34:148-59.

15. Lumpkin EA, Caterina MJ. Mechanisms of sensory transduction in the skin. Nature 2007;445:858-65.

16. Denda M, Sokabe T, Fukumi-Tominaga T, Tominaga M. Effects of skin surface temperature on epidermal permeability barrier homeostasis. J Invest Dermatol 2007;127:654-9.

17. DeLeo JA. Basic science of pain. J Bone Joint Surg [Am] 2006;88:58-62.

18. Hwang-Bo G, Lee JH. Effects of kinesio taping in a physical therapist with acute low back pain due to patient handling: a case report. Int J Occup Med Environ Health 2011;24:320-3.
19. Dupont WD, Plummer WD Jr. Power and sample size calculations. A review and computer program. Control Clin Trials 1990;11:116-28.

20. Dupont WD, Plummer WD Jr. Power and sample size calculations for studies involving linear regression. Control Clin Trials 1998;19:589-601.

21. Available from: https://www.randomizer.org

22. Tousignant M, Poulin L, Marchand S, Viau A, Place C. The Modified-Modified Schober Test for range of motion assessment of lumbar flexion in patients with low back pain: a study of criterion validity, intra- and inter-rater reliability and minimum metrically detectable change. Disabil Rehabil 2005;27:553-9.

23. Fairbank JC, Couper J, Davies JB, O'Brien JP. The Oswestry low back pain disability questionnaire. Physiotherapy 1980;66:271-3.

24. Parreira Pdo C, Costa Lda C, Takahashi R, Hespanhol Junior LC, Luz Junior MA, Silva TM, et al. Kinesio taping to generate skin convolutions is not better than sham taping for people with chronic non-specific low back pain: a randomised trial. J Physiother 2014;60:90-6.

25. Parreira Pdo C, Costa Lda C, Hespanhol LC Jr, Lopes AD, Costa LO. Current evidence does not support the use of Kinesio Taping in clinical practice: a systematic review. J Physiother 2014;60:31-9.

26. Mostafavifar M, Wertz J, Borchers J. A systematic review of the effectiveness of kinesio taping for musculoskeletal injury. Phys Sportsmed 2012;40:33-40.

27. Morris D, Jones D, Ryan H, Ryan CG. The clinical effects of Kinesio ${ }^{\otimes}$ Tex taping: A systematic review. Physiother Theory Pract 2013;29:259-70.

28. Kalron A, Bar-Sela S. A systematic review of the effectiveness of Kinesio Taping--fact or fashion? Eur J Phys Rehabil Med 2013;49:699-709.

29. Nelson NL. Kinesio taping for chronic low back pain: A systematic review. J Bodyw Mov Ther 2016;20:672-81.

30. Kelle B, Güzel R, Sakallı H. The effect of Kinesio taping application for acute non-specific low back pain: a randomized controlled clinical trial. Clin Rehabil 2016;30:997-1003.

31. Kachanathu SJ, Alenazi AM, Seif HE, Hafez AR, Alroumim MA. Comparison between Kinesio Taping and a Traditional Physical Therapy Program in Treatment of Nonspecific Low Back Pain. J Phys Ther Sci 2014;26:1185-8. 